\title{
Kein protektiver Einfluss der Perinatalzeit
}

Fragestellung: Es werden Frauen in psychiatrischer Behandlung in und außerhalb der Perinatalperiode hinsichtlich Suizidtrends, Suizidmethoden und anderen korrelierenden Faktoren verglichen.

Hintergrund: Obwohl die Suizidrate in der Perinatalperiode niedrig ist, ist Suizid bei schwangeren Frauen und Frauen im Wochenbett eine wichtige Todesursache. Es gibt nur wenige Befunde, die als Leitlinie für die Suizidprävention in dieser Gruppe dienen könnten. Während bei Frauen in der Allgemeinbevölkerung in der Postpartalperiode das Suizidrisiko vermindert ist, haben Frauen mit schweren psychischen Erkrankungen postpartal ein erhöhtes Suizidrisiko. Zudem treten in dieser Phase Besonderheiten auf, wie ein häufigeres Absetzen der Medikation, ein plötzlicheres Auftreten oder eine schnelle Verschlechterung einer psychotischen Symptomatik.

Khalifeh H, Hunt IM, Appleby $L$ et al. Suicide in perinatal and non-perinatal women in contact with psychiatric services: 15-year findings from a UK national in quiry. Lancet Psychiatry 2016; 3: $233-42$
Patientinnen und Methodik: Es wurden die zwischen 1997 und 2012 erhobenen Daten des UK National Confidential Inquiry zu Suizid und Mord von Menschen mit psychischen Erkrankungen
(NCISH) ausgewertet. Diese umfassten alle Suizide von Personen ab dem Alter von zehn Jahren, die im vorangegangenen Jahr in psychiatrischer Behandlung waren. Die Studienpopulation umfasste alle Frauen, die durch Suizid verstorben waren; die Frauen, die sich während der Schwangerschaft oder im ersten Jahr postpartum das Leben genommen hatten $(n=98)$ wurden mit den durch Suizid außerhalb der Perinatalperiode in der gleichen Altersklasse verstorbenen Frauen $(n=4.687)$ verglichen.

Ergebnisse: Die Frauen, die infolge Suizids in der Perinatalperiode verstarben, waren jünger und öfter verheiratet, hatten eine kürzere Dauer ihrer psychischen Erkrankung (39\% waren kürzer als ein Jahr erkrankt), häufiger keinen Alkoholmissbrauch in der Vorgeschichte und seltener Gewalterfahrungen als Frauen, die sich außerhalb der Perinatalperiode suizidierten. Die Hälfte der perinatalen Suizidopfer hatte die Diagnose einer Depression (48\%), $13 \%$ einer Schizophrenie und $11 \%$ einer Persönlichkeitsstörung. Frauen in der Perinatalperiode wählten häufiger eine harte Suizidmethode ( $72 \%$ vs. $56 \%$ ) und bei Medikamentenüberdosis seltener psychotrope Substanzen (14\% vs. $39 \%)$. Zwei der 98 Frauen töteten ihr Kind vor dem eigenen Suizid.

Schlussfolgerung: Die Perinatalperiode hat bei psychisch kranken Frauen keinen protektiven Effekt gegen Suizid.

\section{- Kommentar von Barbara Schneider, Köln}

\section{Gelten für psychisch kranke Frauen andere Risikofaktoren?}

Es handelt sich um eine Studie, die auf Daten eines nationalen Registers zu unnatürlichen Todesursachen bei psychisch Kranken basiert und in der zusätzlich die behandelnden Ärzte der durch Suizid verstorbenen Frauen mittels eines Fragebogenverfahrens befragt wurden. Die Untersuchung ist eine der größten Fallserienstudien, in der Frauen, die während der Schwangerschaft und im ersten Jahr nach der Geburt durch Suizid verstorben waren, mit anderen durch Suizid verstorbenen Frauen hinsichtlich diagnostischer und demografischer Merkmale verglichen wurden. Beeinflussende Variablen wurden in den statistischen Verfahren berücksichtigt.

Die Studie zeigte, dass Frauen, die infolge Suizids in der Perinatalperiode verstarben, jünger und häufiger verheiratet waren, kürzere Zeit psychisch krank waren und seltener einen Alkoholmissbrauch in der Vorgeschichte hatten. Fast die Hälfte der Frauen, die sich in der Perinatalperiode das Leben nahm, litt an einer Depression.

Bekannt ist, dass in der Allgemeinbevölkerung Schwangerschaft und die Zeit nach der Geburt eines Kindes einen suizidprotektiven Effekt haben; dies trifft wahrscheinlich seltener für Frauen zu, die bereits psychisch erkrankt sind. Da wenig über ursächliche Faktoren für Suizid in der perinatalen Periode bei psychisch Kranken bekannt ist, hat die Studie eine hohe klinische Relevanz. Insbesondere Frauen mit einer schweren
Depression benötigen in dieser perinatalen Phase engmaschige psychiatrische Behandlung.

Wesentliche Schwächen der Studie sind, dass sich lediglich zirka $60 \%$ der Frauen, die sich perinatal das Leben nehmen, in psychiatrischer Behandlung befinden und letztlich die Ergebnisse nicht auf die Allgemeinbevölkerung (in der andere Risikofaktoren für Suizid in der Perinatalperiode diskutiert werden) übertragen werden können. Dies könnte nur durch epidemiologische Studien (Kohortenstudien oder Fall-Kontroll-Studien) geklärt werden. Jedoch sind aufgrund des seltenen Vorkommens perinataler Suizide diese Studiendesigns zur Untersuchung von Risikofaktoren für perinatale Suizide nicht geeignet.

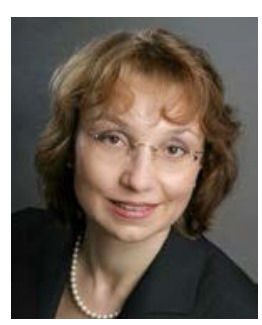

Prof. Dr. med. Barbara Schneider, M.Sc., Köln

LVR-Klinik Köln, Abteilung für Abhängigkeitserkrankungen und Allgemeinpsychiatrie E-Mail:B.Schneider@lvr.de 